\title{
The Roles of Body and Mind in Abstract Thought
}

\author{
Lera Boroditsky (lera@psych.stanford.edu) \\ Department of Psychology, Jordan Hall, Bldg420 \\ Stanford, CA 94305-2130 USA \\ Michael Ramscar (michael@dai.ed.ac.uk) \\ University of Edinburgh, 2 Buccleuch Place \\ Edinburgh, EH8 9LW, Scotland \\ Michael C. Frank (mcfrank@stanford.edu) \\ Department of Psychology, Jordan Hall, Bldg420 \\ Stanford, CA 94305-2130 USA
}

\begin{abstract}
How are we able to think about things we've never seen or touched? We demonstrate that abstract knowledge is built analogically from more experiencebased knowledge. People's understanding of the abstract domain of time, for example, is so intimately dependent on the more experience-based domain of space, that when people make a an air journey or bet on a racehorse, they also unwittingly (and dramatically) change their thinking about time. Further, it appears that abstract thinking is built on representations of more experience-based domains that are functionally separable from those involved directly in sensorimotor experience itself.
\end{abstract}

How are we able to think about things we've never seen or touched? Whether we are theorizing about invisible forces, studying the behaviors of atoms, or trying to characterize the nature of private mental experience, much scientific progress depends on generating new ways of describing and conceptualizing phenomena which are not perceivable through the senses. We face the same problems in everyday life with abstract notions like time, justice, and love. How do we come to represent and reason about abstract domains despite the dearth of sensory information available about them?

One suggestion is that abstract domains are understood through analogical extensions from richer, more experience-based domains (Boroditsky, 2000; Gentner et al., in press; Gibbs, 1994; Holyoak \& Thagard, 1995; Lakoff \& Johnson, 1980, 1999). This experience-based structuring view can be formulated in several strengths. A very strong "embodied" formulation might be that knowledge of abstract domains is tied directly to the body such that abstract notions are understood directly through image schemas and motor schemas (Lakoff \& Johnson, 1999). A milder view might be that abstract knowledge is based on representations of more experience-based domains that are functionally separable from those directly involved in sensorimotor experience.

The studies reported in this paper show that people's understanding of the abstract domain of time is substrated by their knowledge and experiences in the more concrete domain of space $^{1}$. In fact, people's representations of time are so intimately dependent on space that when people engage in particular types of spatial thinking (e.g., embarking on a train journey, or urging on a horse in a race), they unwittingly also change how they think about time. Further (and contrary to the very strong embodied view), it appears that abstract thinking is built on representations of more experience-based domains, and not necessarily on the physical experience itself.

Suppose you are told that next Wednesday's meeting has been moved forward two days. What day is the meeting now that it has been rescheduled? The answer to this question depends on how you choose to think about time. If you think of yourself as moving forward through time (the ego-moving perspective), then moving a meeting "forward" is moving it further in your direction of motion-that is, from Wednesday to Friday. If, on the other hand, you think of time as coming toward you (the time-moving perspective), then moving a meeting "forward" is moving it closer to you - that is, from Wednesday to Monday (Boroditsky, 2000; McGlone \& Harding, 1998; McTaggart, 1908). In a neutral context, people are equally likely to think of themselves as moving through time as they are to think of time as coming toward them, and so are equally

\footnotetext{
${ }^{1}$ This paper only examines one aspect of how people think about time. The domain of time comprises an incredibly complex, heterogeneous and sophisticated set of representations, and much more investigation will be necessary to properly characterize the domain as a whole.
} 
likely to say that the meeting has been moved to Friday (the ego-moving answer) as to Monday (the timemoving answer) (Boroditsky, 2000; McGlone \& Harding, 1998).

But where do these representations of time come from? Is thinking about moving through time based on our more concrete experiences of moving through space? If so - if representations of time are indeed tied to representations of space - then getting people to think about space in a particular way should also influence how they think about time.

\section{Study 1}

To investigate the relationship between spatial thinking and people's thinking about time, we asked 239 Stanford undergraduates to fill out a one-page questionnaire that contained a spatial prime followed by the ambiguous "Next Wednesday's meeting..." question described above. The spatial primes (shown in Figure 1) were designed to get people to think about themselves moving through space on an office-chair (see Figure 1a), or of making an office-chair come toward them through space (see Figure 1b). In both cases, participants were asked to imagine how they would "manuever the chair to the X," and to "draw an arrow indicating the path of motion." The left-right orientation of the diagrams was counterbalanced across subjects. Immediately after subjects completed the spatial prime, they were asked the ambiguous "Next Wednesday's meeting has been moved forward two days" question described above. We were interested in whether subjects would think differently about time right after imagining themselves as moving through space, or imagining things coming toward them.

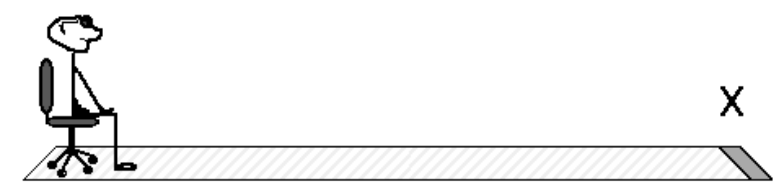

Figure 1a: Spatial prime used in Study 1. Participants were given the following instructions "Imagine you are the person in the picture. Notice there is a chair on wheels, and a track. You are sitting in the chair. While sitting in the chair, imagine how you would manuever the chair to the $\mathbf{X}$. Draw an arrow indicating the path of motion."

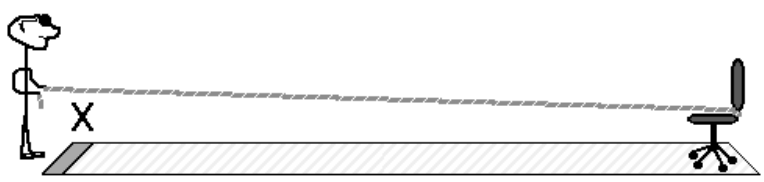

Figure 1b: Spatial prime used in Study 1. Participants were given the following instructions "Imagine you are the person in the picture. Notice there is a chair on wheels, and a track. You are holding a rope attached to the chair. With the rope, imagine how you would manuever the chair to the $\mathbf{X}$. Draw an arrow indicating the path of motion."

As predicted, people used primed spatial information to think about time. Subjects primed to think of objects coming toward them through space, were much more likely to think of time as coming toward them (67\% said Wednesday's meeting had moved to Monday), than they were to think of themselves as moving through time (only 33\% said the meeting has moved to Friday). Subjects primed to think of themselves as moving through space showed the opposite pattern (only $43 \%$ said Monday, and $57 \%$ said Friday), chi-square $=13.3, \quad \mathrm{p}<.001$. It appears that people's thinking about time is indeed tied to their spatial thinking. This raises a further question: do people unwittingly change their thinking about time during everyday spatial experiences and activities (not just when processing specially designed spatial primes in a laboratory setting)?

\section{Study 2: The Lunch-line}

To investigate the relationship between spatial experience and people's thinking about time, we asked 70 people waiting in a lunch-line the ambiguous question about Wednesday's meeting described above. The lunch-line was for a café in the basement of Stanford's Psychology department. The line is usually about 50 meters long, but moves quickly with a waiting time of about 10 minutes. After participants answered our ambiguous question, we asked them how long they felt they had waited in line, and also recorded which quartile of the line they were in when interviewed. This second index served as an objective measure of how much forward motion in line people had experienced (with people furthest along in line having experienced the most motion). We were interested in whether the spatial experience of moving forward in a line would make people more likely to also think of themselves as moving forward in time (as opposed to thinking of time as coming toward them).

As predicted, the further along in line people were (the more forward spatial motion they had experienced), the more likely they were to also think of themselves as moving through time (to say the meeting had been moved to Friday), r=.33 , $\mathrm{p}<.005$ (see Figure 2). People's estimates of their waiting time were also predictive of their answers to the question about next Wednesday's meeting $(\mathrm{r}=.26, \mathrm{p}<.05)$. Interestingly, 
people's estimates of their waiting time were less predictive of their answer to the "Next Wednesday's meeting..." question than was their spatial position in line. When the effect of spatial position was controlled for, people's estimates of their waiting time were no longer predictive of their answers to the ambiguous question about time, $\mathrm{r}=.05, \mathrm{p}=.67$. It appears that spatial position in line (and hence the amount of forward spatial motion that a person had just experienced) was the best predictor of their thinking about time.

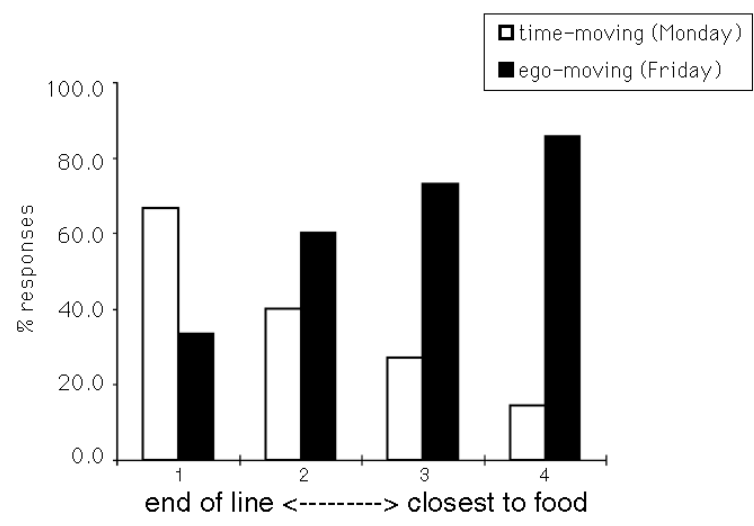

Figure 2: Responses of 70 people waiting in a lunchline. Responses are plotted by position in line (from the end quartile of the line to the quartile closest to the food). The further along in line people were (and hence the more forward spatial motion they had experienced),

the more likely they were to take the ego-moving perspective on time (say that next Wednesday's meeting has been "moved forward" to Friday).

In the next study we were interested in whether spatial motion per se was necessary, or whether simply thinking about or anticipating a journey would be enough to influence how people think about time.

\section{Study 3: The Airport}

To investigate the relationship between spatial experience and people's thinking about time, we asked 333 visitors to San Francisco International Airport the ambiguous question about Wednesday's meeting described above. After the participants answered, we asked them whether they were waiting for someone to arrive, waiting to depart, or had just flown in. We were interested in two things: (1) whether a lengthy experience of moving through space would make people more likely to take the ego-moving perspective on time (think of themselves as moving through time as opposed to thinking of time as coming toward them), and (2) whether the actual experience of motion was necessary to change one's thinking about time, or if just thinking about motion was enough.

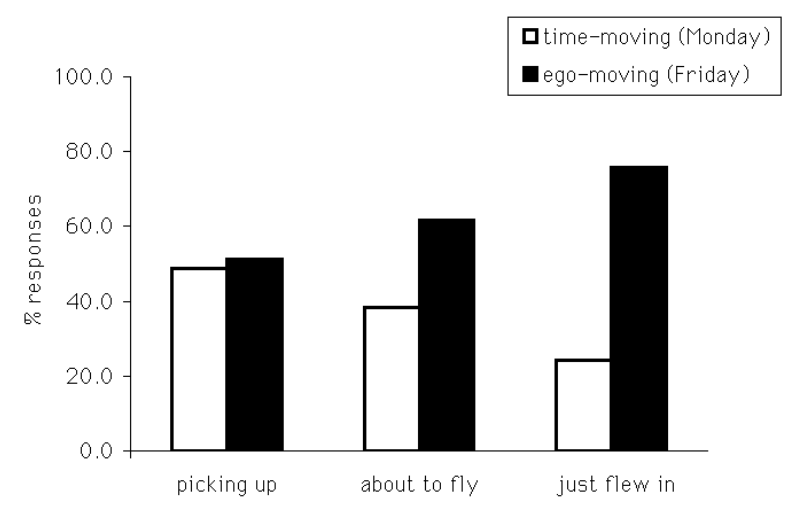

Figure 3: Responses of 333 people queried at the airport. People who had just flown in were most likely

to produce an ego-moving response (say that next

Wednesday's meeting has been "moved forward" to Friday).

As shown in Figure 3, people who had just flown in were much more likely to take the ego-moving perspective (think of themselves as moving through time and answer Friday) (76\%) than people who were just waiting for someone to arrive $(51 \%), \chi^{2}=14.3$, $\mathrm{p}<.01$. Further, even people who hadn't yet flown, but were only waiting to depart were already more likely to think of themselves as moving through time (62\%), $\chi^{2}=4.3, p<.05$ (when compared to people waiting for someone to arrive). This set of findings suggests that (1) people's ideas about time are indeed intimately related to their representations of space, and (2) just thinking about spatial motion is sufficient to change one's thinking about time. But this also raises an interesting question: why were people who had just flown in more likely to take an ego-moving perspective than people who were only about to depart? Was it because they had spent more time actually moving through space, or was it just because they had had more time to think about it?

\section{Study 4: The Train}

To investigate this further, we posed the ambiguous question about Wednesday's meeting to 219 patrons of CalTrain (a train-line connecting San Francisco and San Jose). Of these, 101 were people waiting for the train, and 118 were passengers actually on the train. All of them were seated at the time that they were approached by the experimenter. After participants answered our question, we asked them about how long they had been waiting for (or been on) the train, and how much further they had to go. Participants indicated their 
waiting/travel times using a multiple-choice questionnaire.

First, we found that both people waiting for the train and people actually riding on the train were more likely to take the ego-moving perspective $(63 \%)$ than the time-moving perspective $(37 \%), \chi^{2}=13.9, \mathrm{p}<.01$. Interestingly, the data from people waiting for the train didn't look any different from those of people actually on the train $(61 \%$ and $64 \%$ ego-moving response respectively), suggesting that it is not the experience of spatial motion per se, but rather thinking about spatial motion that underlies our representation of time.

We then examined people's responses based on how long they had been waiting for the train (see Figure 4). The longer people sat around thinking about their journey, the more likely they were to take the egomoving perspective for time. People who had waited less than a minute were equally likely to think of themselves as moving through time as they were to think of time as coming toward them. People who had had five minutes of anticipating their journey, were much more likely to take the ego-moving perspective on time $(68 \%), \chi^{2}=4.5, \mathrm{p}<.05$ (when compared to people waiting less than a minute).

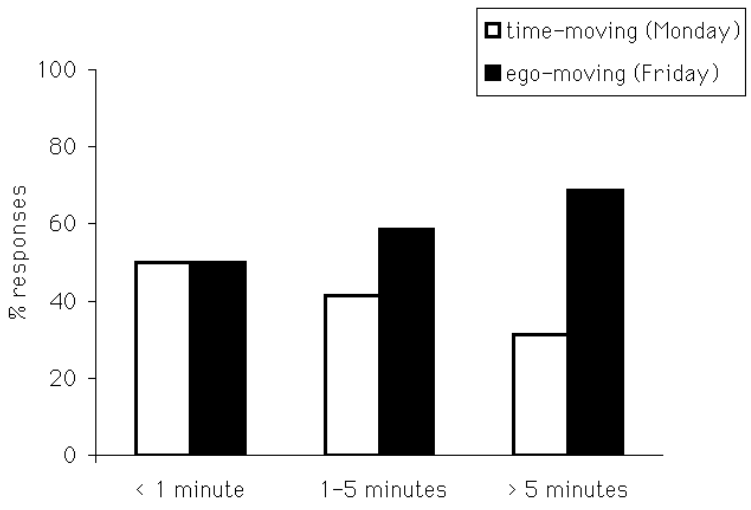

Figure 4: Responses of 101 people waiting for the train plotted by time spent waiting. The more time people had to anticipate their journey, the more likely they became to adopt the ego-moving perspective on time (say that next Wednesday's meeting has been "moved forward" to Friday).

Finally, we analyzed the responses of people on the train based on whether they had answered our ambiguous time question at the beginning, middle, or at end of their journey. We conjectured that people should be most involved in thinking about their journey when they had just boarded the train, or when they were getting close to their destination. In the middle of their journey, people tend to relax, read, talk loudly on cell- phones, and otherwise mentally disengage from being on the train.

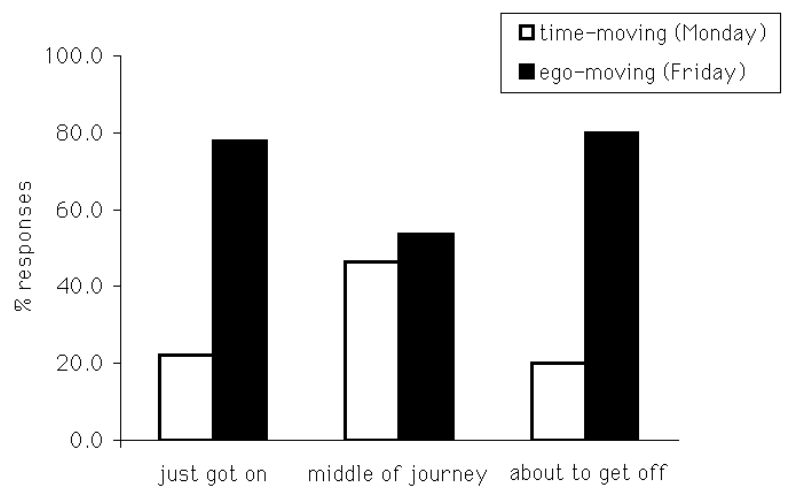

Figure 5: Responses of 118 passengers on the train plotted by point in journey. People became much more likely to adopt the ego-moving perspective for time (say that next Wednesday's meeting has been "moved forward" to Friday) when they were most engaged in thinking about their spatial journey (at the beginnings and ends of the trip). In the middle of their journey, people were about equally likely to adopt the egomoving perspective (say the meeting has been "moved forward" to Friday) as the time-moving perspective (say the meeting has been "moved forward" to Monday).

Amazingly, people's biases for thinking about time mimicked this pattern of engaging and disengaging from spatial thinking perfectly (see Figure 5). Within five minutes of getting on the train, people were very likely to take the ego-moving perspective on time (78\%), $\chi^{2}=6.38, \mathrm{p}<.02$ (when compared to people in the middle of their journey). People were also very likely to take the ego-moving perspective when they were within ten minutes of arriving at their destination $(80 \%), \chi^{2}=5.63$, $\mathrm{p}<.02$ (when compared to people in the middle of their journey). Passengers in the middle of their journey, however, showed no ego-moving bias. They were just as likely to think of themselves as moving through time $(54 \%)$, as they were to think of time as coming toward them (46\%). Once again it appears that people's thinking about time is substrated by thinking about spatial motion and not necessarily by the experience of motion itself. Although all three groups of passengers were having the same physical experience (all were simply sitting on the train), the two groups that were most likely to be involved in thinking about their journey showed the most change in their thinking about time.

\section{Study 5: The Race-Track}

So far, we have only looked at people who themselves were moving or planning to move. Could thinking 
about spatial motion have a similar effect even when people are not planning any of their own motion? To investigate this question, we asked the "Next Wednesday's meeting..." question of 53 visitors to the Bay Meadows racetrack. We predicted that the more involved people were in the forward motion of the racehorses, the more likely they would also be to take the ego-moving perspective on time (and say that the meeting has been moved to Friday). After asking people the question about next Wednesday's meeting, we also asked them how many races they had watched that day and how many races they had bet on. Both indices turned out to be good predictors of people's answers to the "Next Wednesday's meeting..." question. As shown in Figure 6, people who hadn't bet on any races were as likely to think of themselves as moving through time (50\% said Friday), as they were to think of time as coming toward them (50\% said Monday). In contrast, people who had bet on 3 races or more were three times more likely to think of themselves as moving through time ( $76 \%$ said Friday) than they were to think of time as coming toward them (24\% said Monday), \%), $\chi^{2}=12.39, \mathrm{p}<.01$ (when compared to people who hadn't bet on any races). It appears that simply thinking about forward motion (willing a horse towards a finish line, as opposed to actually planning to go somewhere yourself) is enough to change people's thinking about time.

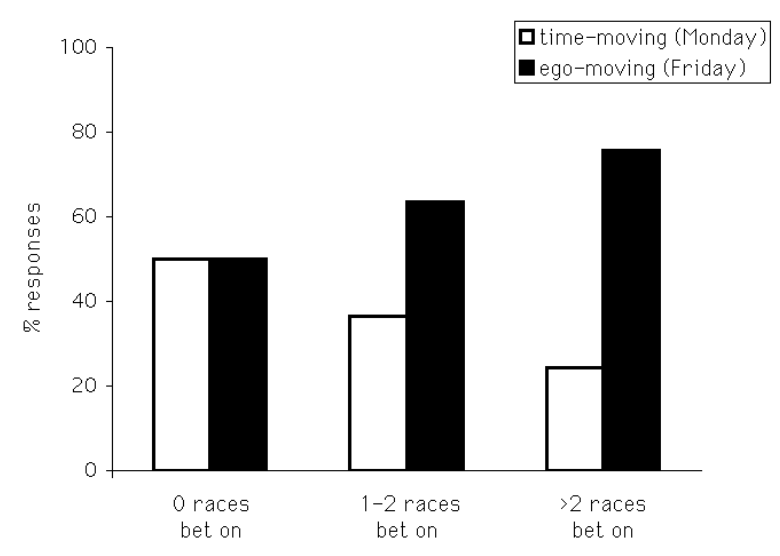

Figure 6: Responses of 53 visitors to the racetrack plotted by number of races bet on. People who had bet on more races (and so were more involved in the forward motions of the racehorses) also became much more likely to adopt the ego-moving perspective for time (say that next Wednesday's meeting has been "moved forward" to Friday).

\section{Study 6: The Office-Chair Rodeo}

Thus far we have shown that people's thinking about spatial motion is a good predictor of their thinking about time and that actual spatial motion is not necessary. A further question is whether actual motion is sufficient to influence people's thinking about time even in the absence of involved spatial thinking.

To try to address this question, we designed a realmotion version of Study 1 (see Figure 1). We set up a $25 \mathrm{ft}$ track outside of the Stanford University Bookstore and invited students to participate in an "office-chair rodeo." Half of the participants were asked to ride an office chair from one end of the track to the other (the ego-moving prime), and half were asked to rope the chair in from the opposite end of the track (the timemoving prime) (see Figure 1 for a diagram). The track was marked out in the asphalt using colored masking tape, with one end of the track marked in red, and one in yellow. Seventy-eight Stanford undergraduates participated in the study in exchange for lollipops. The verbal instructions were the same in both conditions. Participants riding the chair sat in an office-chair at one end of the track and were asked to "maneuver the chair to the red/yellow line" (whichever was at the opposite end of the track). Participants roping the chair were given a rope that was connected to the office-chair at the opposite end of the track and were likewise instructed to "maneuver the chair to the red/yellow line" (whichever was where the participant was standing).

Immediately after the participant completed the motion task (either riding or roping the chair), they were asked the question about next Wednesday's meeting. Interestingly, performing these spatial motion tasks had no effect on subjects' thinking about time. People riding the chair (actually moving through space), were as likely to think of themselves as moving through time $(55 \%$ said the meeting would be on Friday), as were people roping the chair (actually making and object move toward them) (58\% said the meeting would be on Friday). It appears that just moving through space, is not sufficient to influence people's thinking about time. This finding is especially striking when compared to the findings of Study 1 where just thinking about spatial motion (in the absence of any actual motion) was enough to influence people's thinking about time (see also Boroditsky, 2000).

\section{Discussion}

Taken together these studies demonstrate the intimate relationship between abstract thinking and more experience-based forms of knowledge. People's thinking about time is closely linked to their spatial thinking. 
When people engage in particular types of spatial thinking (e.g., thinking about their journey on a train, or urging on a horse in a race), they also unwittingly and dramatically change how they think about time. Further, and contrary to the very strong embodied view, it appears that this kind of abstract thinking is built on representations of more experience-based domains that are functionally separable from those directly involved in sensorimotor experience itself.

A further question is how do these relationships between abstract and concrete domains come about in the first place? Surely, some relationships come from correspondences that can be observed in experience. For example, progression in space and time are often correlated -- movements that are longer spatially are also likely to take a longer amount of time. These simple correspondences in experience can then be amplified and built on by language. People often use metaphors from more experienced-based domains to talk about more abstract domains, and often these metaphors go beyond what can be observed in experience. This means that some abstract knowledge might be constructed and shaped by language. In fact, this turns out to be the case. For example, English and Mandarin speakers use different spatial metaphors to talk about time, and this difference in language leads to important differences in the way the two groups think about time (Boroditsky, in press). It follows that to properly characterize abstract thought, it will be important to look not only at what comes from innate wiring and physical experience, but also at the ways in which languages and cultures have allowed us to go beyond these to make us smart and sophisticated as are.

\section{Acknowledgments}

The authors would like to thank Amy Jean Reid, Justin Weinstein, and Webb Phillips for their heroic feats of data collection. Correspondence and requests for materials should be addressed to Lera Boroditsky (email: lera@psych.stanford.edu).

\section{References}

Boroditsky, L. (2000). Metaphoric structuring: Understanding time through spatial metaphors. Cognition, 75(1), 1-28.

Gentner, D., Bowdle, B., Wolff, P., \& Boronat, C. (in press). Metaphor is like analogy. In Gentner, D., Holyoak, K. J., \& Kokinov, B. N. (Eds.) (in press). The analogical mind: Perspectives from cognitive science. Cambridge, MA: MIT Press.

Gibbs, R.J. (1994). The poetics of mind: Figurative thought, language, and understanding. New York, Cambridge University Press.
Holyoak, K.J. \& Thagard, P. (1995). Mental leaps: Analogy in creative thought. Cambridge, The MIT Press.

Lakoff, G. \& Johnson, M. (1980). Metaphors we live by. University of Chicago Press, Chicago.

Lakoff, G. \& Johnson, M. (1999). Philosophy in the Flesh: The Embodied Mind and Its Challenge to Western Thought. Basic Books, New York.

McGlone, M.S. \& Harding, J.L. (1998). Back (or forward?) to the future: the role of perspective in temporal language comprehension. Journal of Experimental Psychology: Learning Memory and Cognition, 24(5), 1211-1223.

McTaggart, J. (1908). The Unreality of time. Mind, $17,457-474$. 